\title{
A Low Cost Methane Absorption FUELING SYSTEM IN WIRELESS SENSOR NETWORKS USING SBC-MS
}

\author{
A.Rehash Rushmi Pavitra ${ }^{1}$ and Dr. E. Srie Vidhya Janani ${ }^{2}$ \\ 1,2 Department of Computer Science and Engineering, \\ Anna University Regional Campus, Madurai
}

\begin{abstract}
The isolated accessible Methane $\left(\mathrm{CH}_{4}\right)$ Absorption Fueling System (MAFS) is matured based on the mechanics of Wireless Sensor Network (WSN) comprised of gas sensing capable motes complementing a MAFS-WSN. Discrete routing protocols have been designed earlier for data collection in both compatible and divergent networks. This research presents a novel Scheduling based Clustering (SBC) with Mobile Sink (MS) strategy (SBC-MS) which supplements data collection in MAFS-WSN. The SBC-MS strategy attempts to exploit the vital parameters of energy and distance in selecting the appropriate cluster head that well suits $M A F S-W S N$ in reliable gas detection. The MSs are exploited to reduce the energy expenditure in data communication. Extensive experimentations have been carried out with the proposed $S B C-M S$ to ensure the QOS of MAFS-WSN in terms of schedulability and reliability. The simulation results prove that $S B C-M S$ outperforms the earlier clustering technique $M-L E A C H$ in terms of network lifetime, energy consumption, end-to-end delay and data rate.
\end{abstract}

\section{KEYWORDS}

Methane Absorption Fueling system, Gas Sensing Capable Motes, Mobile Sinks, Scheduling and Clustering.

\section{INTRODUCTION}

The Wireless Sensor Networks (WSNs) endeavor solitary benefits and adaptability in premises of low-power and low-cost deployment in variety of applications which in turn has facilitated the originator to design self-governing sensors [12]. In recent years a variety of air pollution monitoring applications finds their way feasible through WSNs. The ambient air monitoring sensors greatly complement towards a systematic monitoring of air quality. The concentration of Carbon Dioxide $\left(\mathrm{CO}_{2}\right)$ in MAFS is quantified by spectroscopic sensors as Non-Dispersive Infra-Red (NDIR) sensors. The level of $\mathrm{CH}_{4}$ is deliberated in terms of Parts Per Million (PPM) such sensors are often used as gas detectors.

\section{NDIR $\mathrm{CO}_{2}$ Gas Sensor and Methane Sensor (TGS2611-E)}

A light weight spectroscopic sensor termed to be a Non-Dispersive Infra-Red (NDIR) sensor quantifies the gas absorption of radiation at an acknowledged wavelength. The cramped size semiconductor type sensors possess high selectivity of methane gas ensure low cost and low power consumption. The MAFS-WSN works on the principle of transformation of carbon dioxide $\left(\mathrm{CO}_{2}\right)$ into methane $\left(\mathrm{CH}_{4}\right)$ over a catalyst, which in 
turn can be precisely recycled as a fuel. Methane (odorless) is an essential intention gas owing to its immense combination in natural gas [13]. The NDIR $\mathrm{CO}_{2}$ Gas Sensors are also capable of identifying and associating themselves to form a network confined for air quality monitoring.

\section{RELATED WORK}

WSNs contribute an outstanding, simple to set up, and economical solution thereby favoring a time automated monitoring of the atmosphere. Consequently, a variety of atmosphere monitoring systems have been proposed. A real time pollution monitoring System [1] employs nodes with fixed gas sensors confined for air quality monitoring. Also, the multi-hop data aggregation algorithm exploits the sensor data to generate overall network statistics together ensuring a feasible view of the network.

With relevance to the Mobile Ad Hoc Network (MANET) routing algorithm [8], a set of mobile nodes deployed in the network seem to figure out the status of gas in contrasting field together alerting the smart phone users. The algorithm not only measures the level of air pollution over cities but also ensures the traceability of sender and receiver across the cloud.

The air quality monitoring is identified to be better in terms of data classification [9] with decision tree algorithm. The algorithm isolates a set of data into predefined classes thereby defining a tree structure for accurate monitoring of air quality level.

The monitoring of greenhouse gases has been made possible through a solar powered unmanned aerial vehicle [2].

The gas detectors seem to find their utility in Wireless Gas Sensor Network (WGSN) to detect and monitor harmful gases in utility areas and industries [3]. The WGSN not only encounters the leakage of harmful gases but also approximates the concentration they amount to in the atmosphere thereby setting up a corresponding audio-visual alarm.

By combining wireless sensor network (WSN) and Global System for Mobile (GSM) technology a low-power consumption monitoring system complements to detect gas leakage ensuring home security [4].

The minimum concentration of Methane in air that amounts to ignition has been analyzed with reference to a key parameter Lower Explosive Limit (LEL) [5].

WSN stationed with Meshlium gateway [6] complements towards $\mathrm{CO}$ and $\mathrm{CO}_{2}$ pollution monitoring. The data communication from gas sensors to server over the Meshlium gateway seems to be efficient.

Continuous Air Quality Monitoring Stations (CAQMS) [7] supplement a custom Information System to store, visualize and analyze the air quality related data which are further presentable in a database.

A Hybrid Spatio-Temporal [HST] scheme is suggested [10] to efficiently forecast air pollution interacting with ubiquitous mobile sensor network. The analyses of existing techniques are laid out in Table 1. 
Table 1. Analysis of existing techniques

\begin{tabular}{|c|c|c|c|c|}
\hline Scheme & Technique & Parameters & Results & Deficiency \\
\hline $\begin{array}{c}\text { Data Aggregation } \\
\text { Algorithm [1] }\end{array}$ & Multi-hop & $\begin{array}{c}\text { Temperature, } \\
\text { Concentration of } \\
\mathrm{CO}_{2}\end{array}$ & $\begin{array}{l}\text { Increased stability } \\
\text { period and strong } \\
\text { output signal }\end{array}$ & $\begin{array}{c}\text { Motes expose a } \\
\text { high power } \\
\text { consumption }\end{array}$ \\
\hline $\begin{array}{l}\text { MANET Routing } \\
\text { Algorithm [8] }\end{array}$ & Static & $\begin{array}{l}\text { Data type, Speed of } \\
\text { transmission, } \\
\text { Coverage of system }\end{array}$ & $\begin{array}{l}\text { Monitoring of } \\
\text { humidity, } \\
\text { temperature and } \\
\text { gas level }\end{array}$ & $\begin{array}{l}\text { High energy } \\
\text { expenditure }\end{array}$ \\
\hline $\begin{array}{l}\text { Classification } \\
\text { Algorithm [9] }\end{array}$ & Chain based & $\begin{array}{c}\text { Accuracy, } \\
\text { sensitivity and } \\
\text { specificity }\end{array}$ & High Accuracy & $\begin{array}{c}\text { High latency } \\
\text { and processing } \\
\text { overhead }\end{array}$ \\
\hline MOX and NDIR [2] & $\begin{array}{l}\text { Statistical } \\
\text { information }\end{array}$ & $\begin{array}{c}\text { Reliable } \\
\text { performance, } \\
\text { Payload constraints }\end{array}$ & $\begin{array}{l}\text { Effective 3D } \\
\text { monitoring }\end{array}$ & $\begin{array}{l}\text { High energy } \\
\text { consumption }\end{array}$ \\
\hline Zigbee Standard [3] & Fixed node & $\begin{array}{l}\text { Optimum Received } \\
\text { signal strength } \\
\text { indication }\end{array}$ & $\begin{array}{c}\text { Better leakage } \\
\text { detection }\end{array}$ & $\begin{array}{l}\text { Decrease in } \\
\text { network } \\
\text { throughput }\end{array}$ \\
\hline $\begin{array}{c}\text { Embedded Logic } \\
{[4]}\end{array}$ & Multi modal & Single bit output & High reliability & $\begin{array}{l}\text { Minimum } \\
\text { network } \\
\text { lifetime. }\end{array}$ \\
\hline Wi-Fi Security [5] & $\begin{array}{c}\text { Statistical } \\
\text { Information }\end{array}$ & $\begin{array}{c}\text { Data quality level, } \\
\text { Data quality } \\
\text { assessment }\end{array}$ & $\begin{array}{c}\text { Optimal data } \\
\text { security and } \\
\text { throughput }\end{array}$ & $\begin{array}{l}\text { Poor event } \\
\text { detection }\end{array}$ \\
\hline $\begin{array}{c}\text { End Device(Meshlium } \\
\text { Gateway) [6] }\end{array}$ & Database & $\begin{array}{c}\text { Perform data } \\
\text { readout, Visualize } \\
\text { the data }\end{array}$ & $\begin{array}{c}\text { Data forwarding } \\
\text { using Zigbee }\end{array}$ & $\begin{array}{c}\text { Dependency } \\
\text { over local } \\
\text { database } \\
\end{array}$ \\
\hline CAQMS [7] & Star topology & Correlation value & $\begin{array}{l}\text { Better system } \\
\text { reliability and } \\
\text { energy autonomy }\end{array}$ & High Latency \\
\hline HST [10] & Grid data & $\begin{array}{l}\text { Accuracy } \\
\text { prediction, } \\
\text { Capacity }\end{array}$ & $\begin{array}{l}\text { Data collection } \\
\text { via Bluetooth }\end{array}$ & $\begin{array}{l}\text { Highly prone to } \\
\text { errors }\end{array}$ \\
\hline
\end{tabular}

\section{SYSTEM MODEL}

Primary Assumptions:

The formal system model is based on some fundamental assumptions on the different network nodes and mechanisms involved.

- $\quad$ Non-Dispersive Infrared Sensors with an appropriate introverted ID to measure $\mathrm{CO}_{2}$.

- $\quad$ SBC-MS strategy to ensure reliable gas detection.

- $\quad$ MS computerized with a non-zero value is assumed to migrate within the network.

\section{NETWORK MODEL}

Initially, three tankers of same size (100 tons) T1, T2 and T3 are deployed in the network field of $600 * 600$ square meter area. The $\mathrm{CO}_{2}$ absorption tanker $\mathrm{T} 1$ absorbs the relevant gas from the atmosphere. The tanker T2 coated with $\mathrm{Zr}$ nanoparticles is responsible for Methane conversion. Subsequently, the tanker T3 is employed to act as the Gas Storage System (GSS) to store $\mathrm{CH}_{4}$. TheNDIR sensor nodes are deployed randomly in the first thereby ensuring a hierarchical clustering phenomenon. A Mobile Sink (MS) is used for the data collection task. Figure 1. demonstrates the network model, where migrates towards the first tanker to collect information on the level of $\mathrm{CO}_{2}$ from the NDIR sensors. 


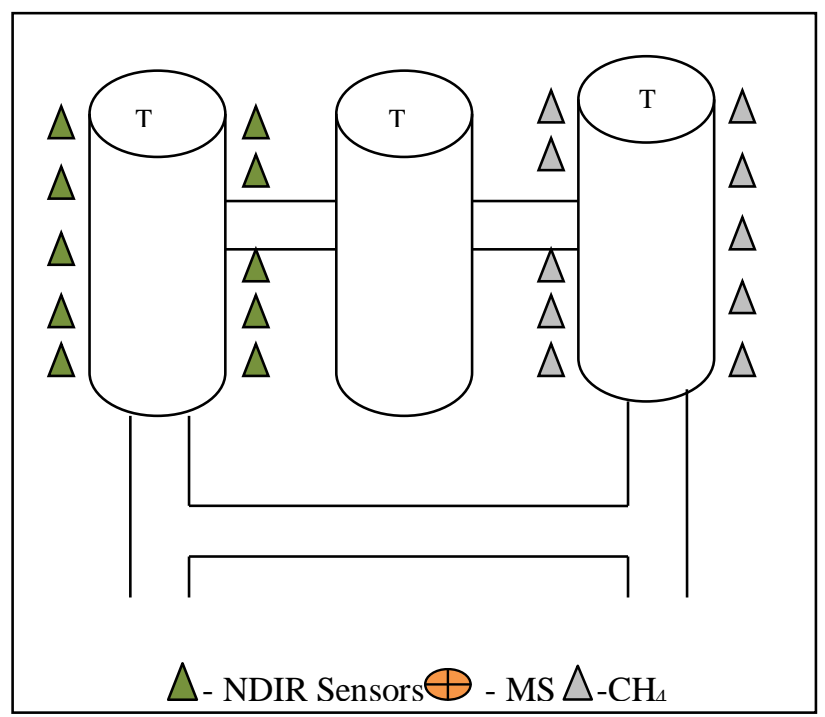

Figure 1. Network Model of MAFS

\section{PROPOSED WORK}

The Scheduling based Clustering with Mobile Sink strategy balances the energy consumption across the network ultimately increasing the lifetime of MAFS-WSN. The data collection strategy with MS reduces direct transmissions from sensors to BS thereby increasing the lifetime of the individual sensors [14]. The relevant information on the level of $\mathrm{CO}_{2}, \mathrm{CH}_{4}$ is sent by the MS to BS over the gateway progressively. BS is deemed to be the system organizer that approximates the level of $\mathrm{CH}_{4}$ in Parts Per Million (PPM) as available in the gas storage system. Figure 2. Shows the key theme for the MAFS-WSN.

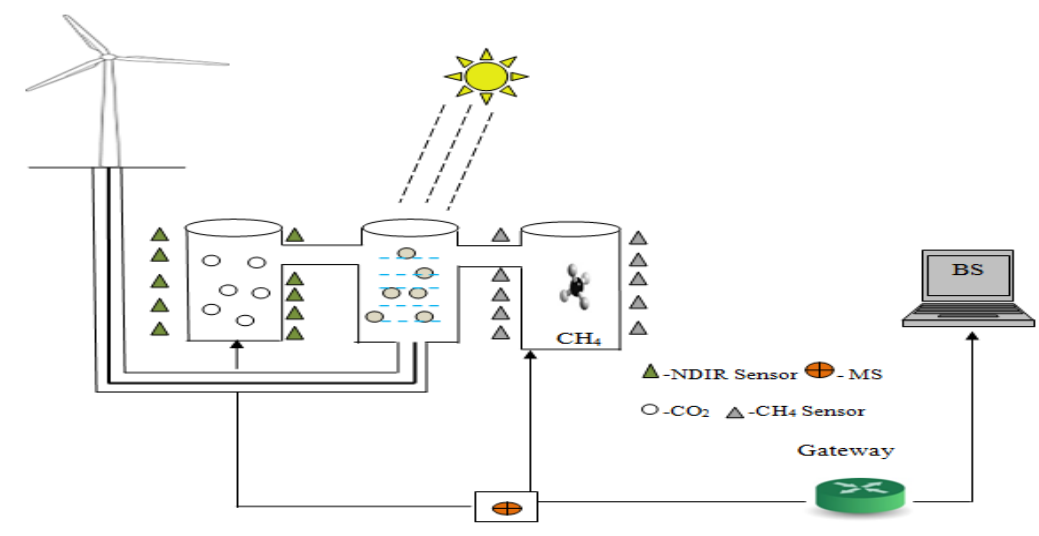

Figure 2. Key theme for the MAFS-WSN

\subsection{Development of the Proposed Mafs}

MAFS system is used for the real time monitoring of greenhouse gases such as $\mathrm{CO}_{2}, \mathrm{CH}_{4}$. The wind turbine converts atmospheric hydrogen into electric power. The first tanker involves a Metal Organic Framework (MOF) coated with $(\mathrm{Ru} / \mathrm{Zr})$ that further complements gas saturation and catalysis to capture $\mathrm{CO}_{2}$.

The second tanker is filled with water. The zirconium atoms yielded through the first tanker dissolves in the water to form Zirconium Dioxide $\left(\mathrm{ZrO}_{2}\right)$ [11]. When the electric energy and $\mathrm{ZrO}_{2}$ fuse they form hydroxide radicals and hydrogen ions to generate hydrogen gas. 


$$
\mathrm{CO}_{2} \underset{3 \mathrm{H}_{2}}{\longrightarrow} \mathrm{CH}_{4}
$$

Subsequently, the combination of $\mathrm{CO}_{2}$ with hydrogen produces $\mathrm{CH}_{4}$ (natural gas). The combination process exploits solar energy to ensure high production of $\mathrm{CH}_{4}$ with a meager amount of catalyst. To illustrate the proposed scheme in detail by dividing them into three phases; (1) $\mathrm{CO}_{2}$ gas monitoring (2) Basis of SBC-MS CH Selection (3) Gas Storage System (4) Data transmission.

\section{2. $\mathrm{Co}_{2}$ Gas Monitoring}

We deploy definite NDIR sensors (non-dispersive infrared) with measuring proposition based

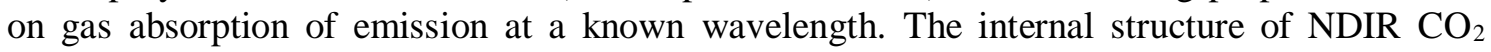
sensor involves an Infrared lamp, a measuring chamber and absorption detector are stated in Figure 3. The difference between the amount of Infrared light among the source and the detector is measured. This difference is directly proportional to the number of carbon dioxide molecules present in the gas [15]. The specifications of an NDIR sensor are shown in Table 2.

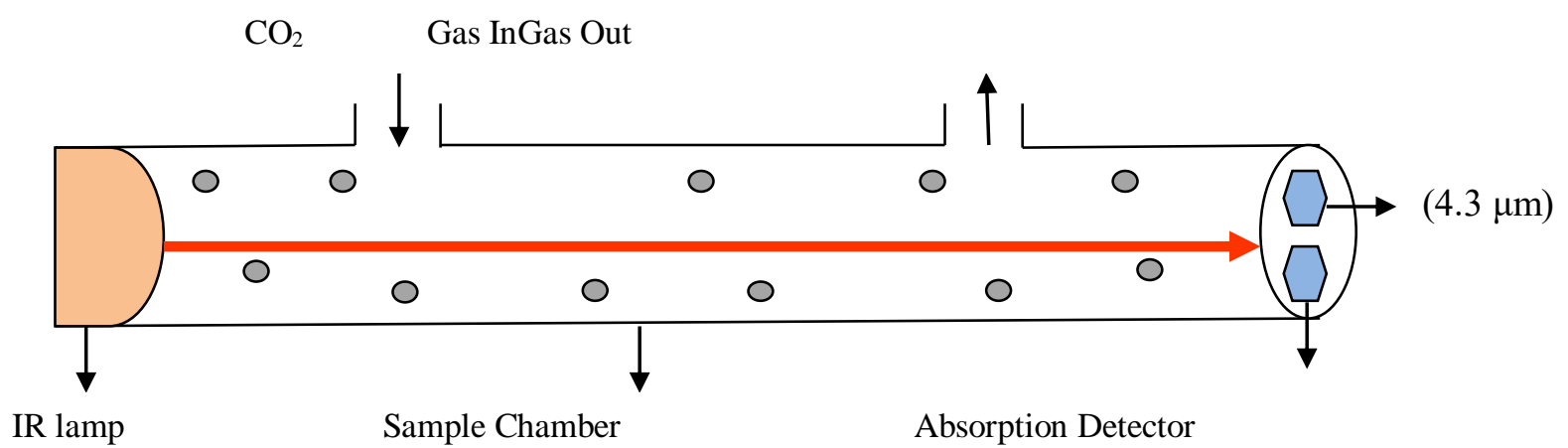

Figure 3. Internal Structure of NDIR $\mathrm{CO}_{2}$ sensor

The $\mathrm{CO}_{2}$ gas molecules inside the chamber can be quantified with the Lambert-Beer's Law.

$$
\mathrm{I}=\mathrm{I}_{0} \times \mathrm{e}^{-\mathrm{kx} 1 \mathrm{x}[\mathrm{co} 2]}
$$

$\mathrm{I}=$ light power intensity after absorption by $\mathrm{CO}_{2}$, measured at the detector $\left(\mathrm{W} \cdot \mathrm{m}^{-2}\right)$

$\mathrm{I}_{0}=$ light power intensity at the source $\left(\mathrm{W} \cdot \mathrm{m}^{-2}\right)$

$\mathrm{k}=$ absorption index of $\mathrm{CO} 2$ at $4.3 \mu \mathrm{m}$ (dimensionless)

$1=$ length of the absorption path $(\mathrm{cm})$

Table 2. NDIR Sensor Specifications

\begin{tabular}{|c|c|}
\hline Parameters & Value \\
\hline Operating Temperature & $0^{0} \mathrm{c}-50^{0} \mathrm{c}$ \\
\hline Storage Temperature & $-30^{0} \mathrm{c}-70^{\circ} \mathrm{c}$ \\
\hline Measurement Range & $0-10000$ PPM (Parts per \\
& million) \\
\hline
\end{tabular}




\subsection{Basis of Sbc-Ms in CH Selection}

\section{CH Selection procedure}

Consider two nodes N1 and N2 with different energy levels. CH selection process is employed to choose an optimal $\mathrm{CH}$ in terms of residual energy and distance from the surface level of the BS. Assuming N1 is selected as a $\mathrm{CH}$ [19], it broadcasts hello message packet to its neighboring sensors within its transmission range. On the other hand, Cluster Member (CM) nodes in a cluster send their data to the corresponding $\mathrm{CH}$. Periodically MS move towards the $\mathrm{CHs}$ collect data and transmit data to the BS, over a gateway.

\subsection{Gas Storage System}

The Gas Storage System is deployed with small size semiconductor type Methane $\left(\mathrm{CH}_{4}\right)$ sensors Figure 4. shows the circuit diagram of Methane $\left(\mathrm{CH}_{4}\right)$ sensors. Initially two input voltage heater voltage $\left(\mathrm{V}_{\mathrm{H}}\right)$ and circuit voltage $\left(\mathrm{V}_{\mathrm{C}}\right)$ is enforced across the sensor element which has a resistance $\left(R_{s}\right)$ between the sensors, electrodes and the load resistor $\left(R_{L}\right)$ connected in series. The $\left(\mathrm{V}_{\mathrm{C}}\right)$ may be applied periodically. The sensor signal $\left(\mathrm{V}_{\mathrm{RL}}\right)$ is calculated eventual as a change in voltage over the $\mathrm{R}_{\mathrm{L}}$. The (Rs) is obtained with relevance to equation (4); The SBCMS strategy discussed earlier is adopted. The specifications of Methane sensor are shown in Table 3.

$$
R_{S=} \frac{V_{C}-V_{R L} \times R_{L}}{V_{R L}}
$$

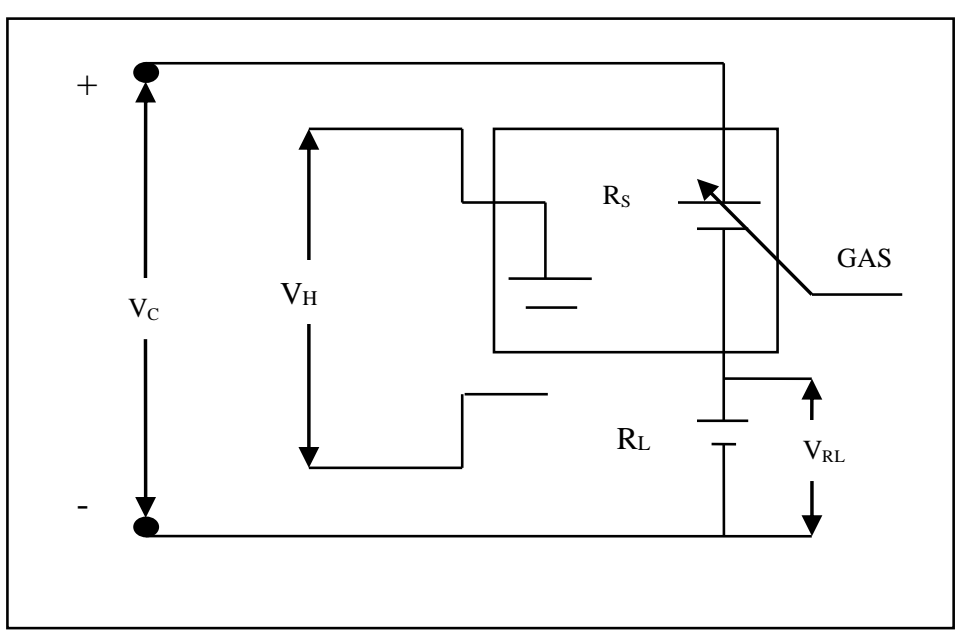

Figure 4. Circuit diagram of Methane Sensor

\begin{tabular}{|c|c|}
\hline Parameters & Value \\
\hline$\left(\mathrm{V}_{\mathrm{C}}\right)$ and $\left(\mathrm{V}_{\mathrm{H}}\right)$ & $5.0 \mathrm{~V} \pm 0.2 \mathrm{~V} \mathrm{AC} / \mathrm{DC}$ \\
\hline Operating \& storage temperature & $-40^{\circ} \mathrm{C} \sim+70^{\circ} \mathrm{C}$ \\
\hline Load resistance $\left(\mathrm{R}_{\mathrm{L}}\right)$ & $(0.45 \mathrm{k} \Omega \mathrm{min})$ \\
\hline
\end{tabular}

Table 3. Methane Sensor Specifications 


\subsection{Data Transmission}

In MAFS-WSN, after node organization and schedule generation, the consequent step is data transmission. Sensor nodes collect data and transfers to $\mathrm{CH}$; $\mathrm{CH}$ sends acknowledgment to the MS. The sink moves towards the $\mathrm{CH}$ and collects data and sends to the BS medium of gateway. The flow chart process is to finding gasconcentration in the network is demonstrated in Figure 5.

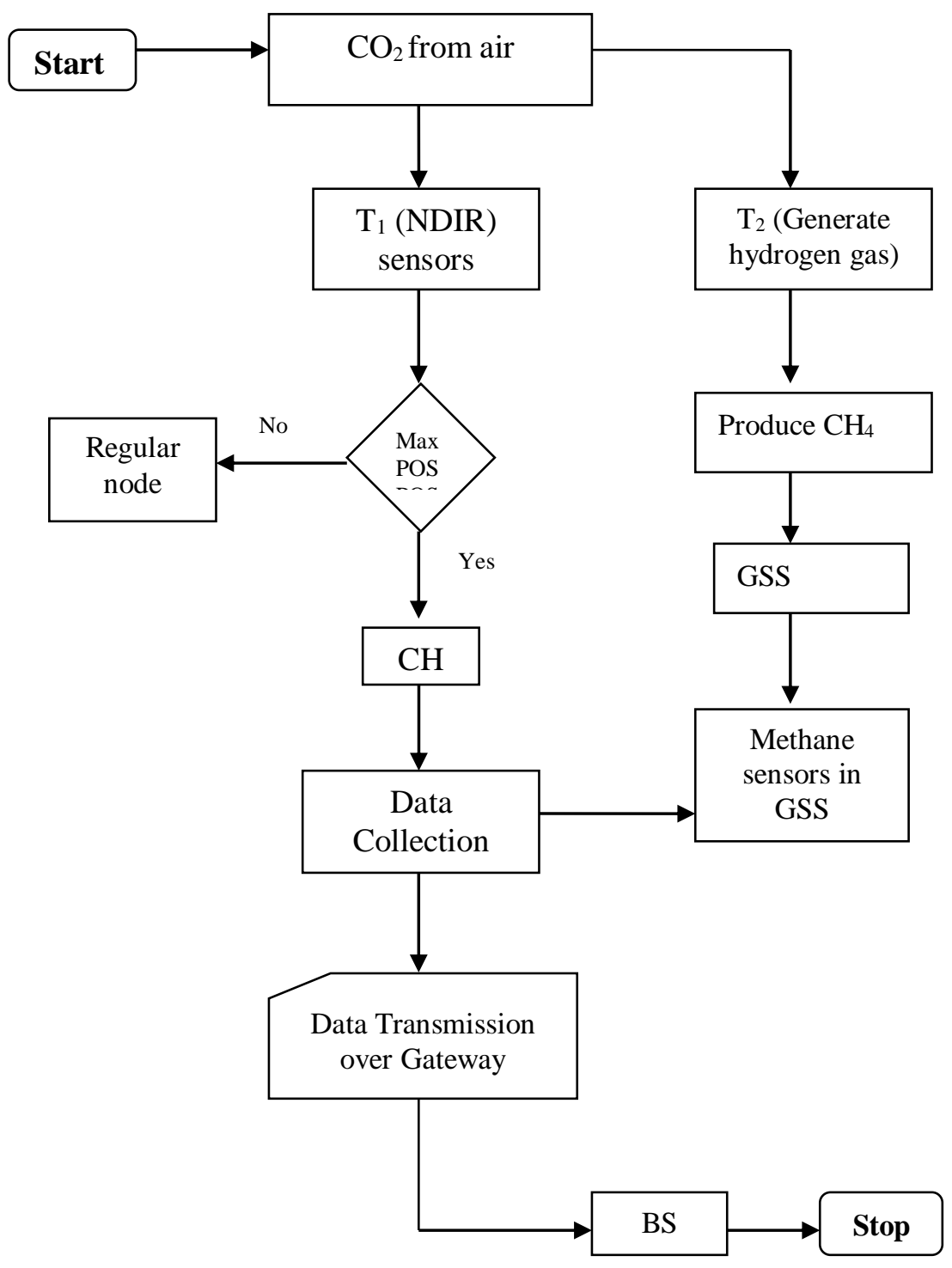

Figure 5. Flow chart of SBC-MS

\section{Simulation Results}

The mathematical analysis was demonstrated using simulations on MATLAB $[17,18]$ in which 100 nodes are deployed randomly in $600 \mathrm{~m}$ x $600 \mathrm{~m}$ region. The initial energy of each node is set to be 5J. A comparative analysis of SBC-MS with the earlier clustering technique MLEACH was performed with the simulation parameters stated in Table 4 and a variation of graphs were plotted which ultimately prove SBC-MS to outperform MLEACH. 
Table 4. Simulation Parameters

\begin{tabular}{|c|c|}
\hline Parameters & Values \\
\hline Network Size & $600 \times 600$ \\
\hline Number of Nodes & 100 \\
\hline Initial Energy & $5 \mathrm{~J}$ \\
\hline Number of Sink & 1 \\
\hline Transmission Range & $25 \mathrm{~m}$ \\
\hline Sink Speed & $5 \mathrm{~m} / \mathrm{s}$ \\
\hline
\end{tabular}

\subsection{Performance Evaluation}

Concentration of $\mathrm{CO}_{2}$ : The results of the $\mathrm{CO}_{2}$ concentration performed with the NDIR sensor. Figure 6. shows the concentration of $\mathrm{CO}_{2}$ in terms of PPM at an operating temperature of $20^{\circ} \mathrm{c}$. The dashed line indicates the amount of $\mathrm{CO}_{2}$ gas in a certain region.

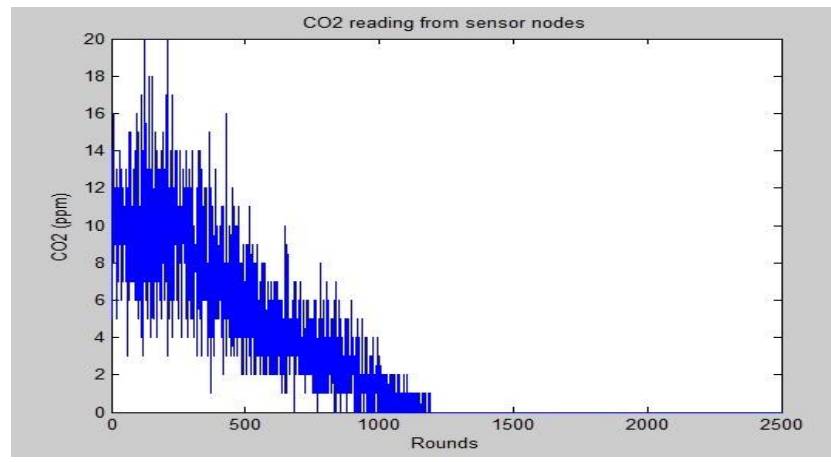

Figure 6. Concentration of $\mathrm{CO}_{2}$

Network Lifetime: Network lifetime is quantified in terms of the total number of nodes alive over different ranges of common rounds. The analysis results prove that all the 100 nodes are alive after the completion of rounds in SBC-MS. Figure 7. compares the proposed SC-MS with the existing MLEACH in terms of network lifetime [16].

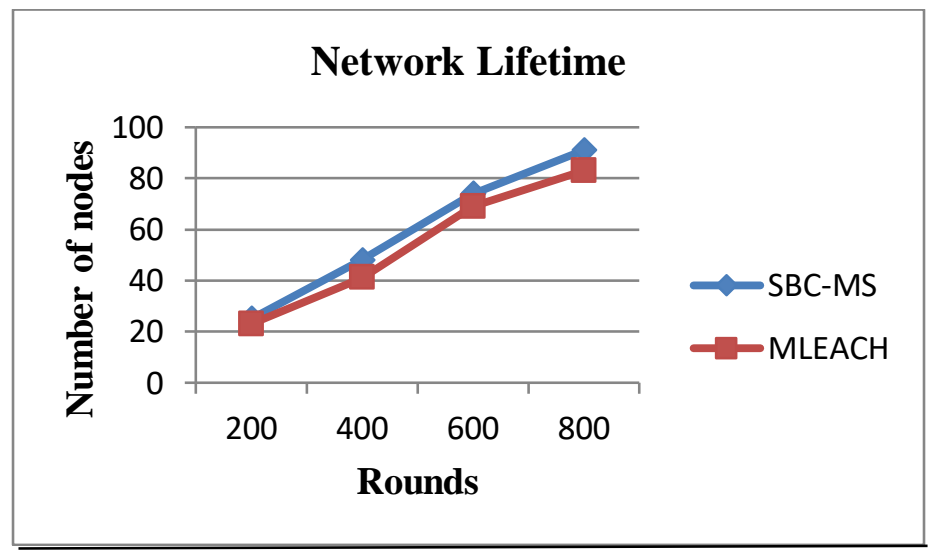

Figure 7. Network Lifetime

End-to-End Delay: The simulation work attempts to quantify end to end delay (in seconds) of the proposed SBC-MS and MLEACH subjected to an increasing number of sensor nodes deployed. The SBC-MS seems to be convincing in terms of delay, when compared to MLEACH is represented in Figure 8 [16]. Since MSs is involved in data collection task, transmission delay 
reduces phenomenally in SBC-MS.

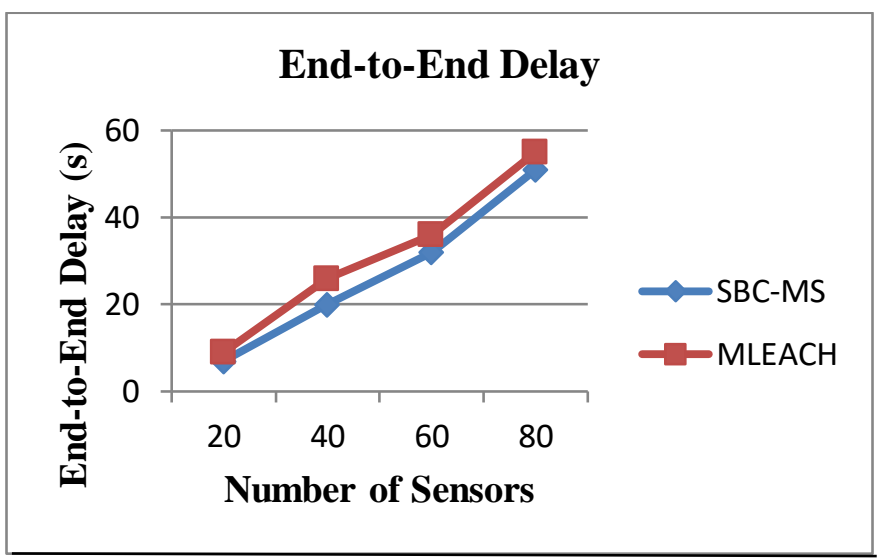

Figure 8. End-to-End Delay

Date Rate: The amount of data transmitted from $\mathrm{CH}$ to MS is quantified in bps as shown in Figure 9 [16]. SBC-MS outperforms MLEACH in terms of data rate thereby avoiding remote transmission.

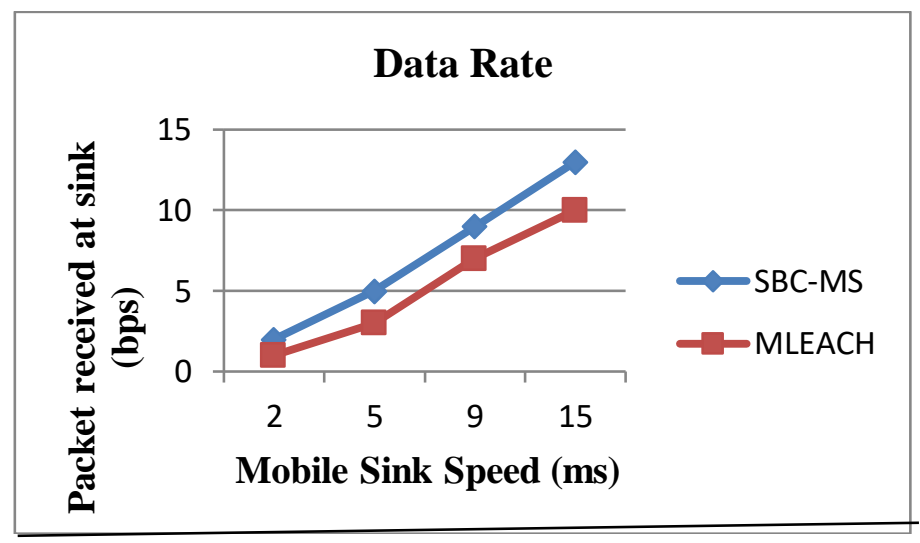

Figure 9. Data Rate

\section{Conclusion}

The fuel system was successfully developed for the direct use of hydrate as a source of fuel for automotive engines. Also, the proposed MAFS-WSN exploits solar energy which seems to be an effective method of hydrate dissociation for automobiles. The modification in the conventional gas transport system reduces the cost of methane extracted from biogas that can further complement to energy independent technologies thereby assisting in utilization of renewable energy. The MAFS-WSN is proven to be better in terms of schedulability and reliability with the proposed SBC-MS algorithm. 


\section{REFERENCES}

[1] Movva Pavani and P.Trinatha Rao, "Real Time Pollution Monitoring Using Wireless Sensor Networks,"Information Technology, Electronics and Mobile Communication Conference (IEMCON), 2016.

[2] Alexander Malaver, Nunzio Motta, Peter Corke and Felipe Gonzalez, "Development and Integration of a Solar Powered Unmanned Aerial Vehicle and a Wireless Sensor Network to Monitor Greenhouse Gases” Sensors, Vol.15, PP. 4072-4096, 2015.

[3] J.Sunil kumar, A.Dileep, "Wireless Gas Sensor Network for Detection and Monitoring of Harmful Gases in Utility Areas and Industries" International Journal of Innovative Engineering Research, Vol. 2, Issue 1, 2014.

[4] B. Shiva Kumar, T. Rama krishnaiah, "GSM technology based Wireless Sensor Network for Gas Monitoring” International Journal of Science, Engineering and Technology Research, Volume 3, Issue 9, September 2014.

[5] Nadime Azizi, Alecia Copeland, John Chun, Matt Ell, Khushbu Kanani, and Zuhair Khoury, "A Study of Methane Sensors in Internet-of-Things Technology" Proceedings of Student-Faculty Research Day, 2016.

[6] M. Udin Harun Al Rasyid, Isbat Uzzin Nadhori and Yodhista Tulus Alnovinda, "CO and CO2 Pollution Monitoring Based on Wireless Sensor Network" IEEE International Conference on Aerospace Electronics and Remote Sensing Technology, 2015.

[7] David Chavez M, River Quispe T, Jorge Rojas M, Andres Jacoby K and Guillermo Garayar L, "A Low-Cost, Rapid-Deployment and Energy-Autonomous Wireless Sensor Network for Air Quality Monitoring” International Conference on Sensing Technology, 2015.

[8] Suganya E, Vijayashaarathi S, "Smart Vehicle Monitoring System for Air Pollution Detection using Wsn” International Conference on Communication and Signal Processing, 2016.

[9] Bambang Sugiarto, Rika Sustika,"Data Classification for Air Quality on Wireless Sensor Network Monitoring System Using Decision Tree Algorithm” International Conference on Science and Technology-Computer, 2016.

[10] Ke Hu, Ashfaqur Rahman, Vijay Sivaraman and Pradeep Ray, "Improving Air Pollution Forecast with Ubiquitous Mobile Sensor Network" International Conference on Ubiquitous and Future Networks (ICUFN), 2014.

[11] R. Lippi, S. C. Howard, H. Barron, C. D. Easton, I. C. Madsen, L. J. Waddington, C.Vogt, M. R. Hill, C. J. Sumby, C. J. Doonan, and D. F. Kennedy, "Highly Active Catalyst for CO2 Methanation Derived from a Metal Organic Framework Template" Journal of Materials Chemistry A, PP.1-9, 2017.

[12] Jun Zheng, Abbas Jamalipour, "Introduction to Wireless Sensor Networks" Institute of Electrical and Electronics Engineers, 2009.

[13] Jasmeen Kaur, Viacheslav I. Adamchuk, Joann K. Whalen and Ashraf A. Ismail, "Development of an NDIR CO2 Sensor-Based System for Assessing Soil Toxicity Using Substrate-Induced Respiration" Sensors, Vol.15, PP. 4734-4748, 2015.

[14] Yongmin Zhang, Shibo He and Jiming Chen,"Near Optimal Data Gathering in Rechargeable Sensor Networks with a Mobile Sink" IEEE Transactions on Mobile Computing, Vol.16, Issue.6, 2017.

[15] Luciano B. Mendes, Nico W. M. Ogink, Nadège Edouard, Hendrik Jan C. van Dooren , Ilda de Fátima F. Tinôco and Julio Mosquera," NDIR Gas Sensor for Spatial Monitoring of Carbon Dioxide Concentrations in Naturally Ventilated Livestock Buildings" Sensors,Vol.15, PP.1123911257, 2015. 
[16] A.Rehash Rushmi Pavitra, E.Srie Vidhya Janani, “A Diplomatic Data Collection in Wireless Sensor Networks using MLEACH Algorithm” International Journal of Computer Sciences and Engineering, Vol.6, Issue.7, PP.2347-2693, 2018.

[17] Onwusuru I, Okolo Chidiebere C, Uka Chukwuma, Ohuabunwa Austine and Nwafor Chibuikem, "Introduction to MATLAB for Researchers and Engineering Students" Journal of Scientific and Engineering Research, Vol.4(6), PP.176-180, 2017.

[18] David Houcque "Introduction to Matlab for Engineering Students", 2005.

[19] Dahlila Putri Dahnil, Yaswant Prasad Singh and Chin Kuan Ho,"Connectivity-Aware and Minimum Energy Dissipation Protocol in Wireless Sensor Networks" International Journal of Distributed Sensor Networks, Vol.9, Issue.7, 2013.

\section{AUTHORS}

A. Rehash Rushmi Pavitra obtained her Bachelor of Engineering Degree in Computer Science and Engineering from Hindusthan College of Engineering and Technology, Coimbatore and received her M.E degree from Anna University Regional Campus, Madurai. She is a Research Scholar carrying out her research in Underwater Wireless Sensor Networks. Her main research interests include Underwater Wireless Sensor Networks with emphasis on Energy Efficiency and Data Aggregation.

Dr.E.Srie Vidhya Jananiis currently working as an Assistant Professor, in the department of Computer Science and Engineering at Anna University Regional Campus, Madurai. Also she obtained his Ph.D degree in Information and Communication Engineering from Anna University Chennai. She has published 15 research papers in reputed International, national journal and conferences. Her current areas of interests are Enhancing Energy Conservation in Wireless Sensor Networks, and Network Security.
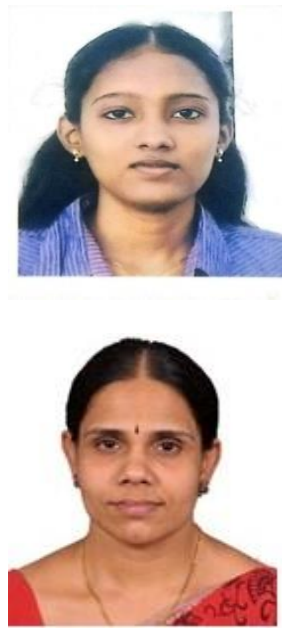